\title{
Practical Use of Lactate Levels in the Intensive Care
}

Journal of Intensive Care Medicine

I-7

(C) The Author(s) 2017

Reprints and permission:

sagepub.com/journalsPermissions.nav DOI: 10.1 |77/08850666/7708563

journals.sagepub.com/home/jic

@SAGE

\section{Eva E. Vink, MD, $P h D^{1,2}$, and Jan Bakker, MD, $P h D^{1,2,3,4}$}

\begin{abstract}
Hyperlactatemia is a strong predictor of mortality in diverse populations of critically ill patients. In this article, we will give an overview of how lactate is used in the intensive care unit. We describe the use of lactate as a predictor of outcome, as a marker to initiate therapy and to monitor adequacy of initiated treatments.
\end{abstract}

\section{Keywords}

lactate, intensive care, prognostication, initiation of treatment

\section{Introduction}

Hyperlactatemia has been associated with morbidity and mortality since the first description in 1843 by Scherer. ${ }^{1}$ The use of lactate as a prognostic tool was first suggested by Broder and Weil more than 120 years later. ${ }^{2}$ They observed that a lactate level $\geq 4 \mathrm{mmol} / \mathrm{L}$ was associated with a $50 \%$ mortality in patients with shock. ${ }^{2}$ More than 30 years later, the mortality associated with this lactate level is similar. ${ }^{3}$ Nowadays, lactate is easily available and routinely measured in most critically ill patients in the intensive care unit (ICU) and emergency department (ED). In this article, we will give an overview of how lactate is used in the ICU. We discuss the use of lactate as a predictor of outcome, as a marker to initiate therapy and to monitor adequacy of initiated treatments.

\section{The Pathophysiology of Lactate}

To fully understand the mechanisms involved in the 3 topics being discussed, it is important to have knowledge on the metabolism of lactate. Lactate exists in the body as 2 stereoisomers: L-lactate and D-lactate. In humans, L-lactate is the dominant isomer that is synthesized and utilized. Accumulation of this isomer represents the vast majority of clinical cases of hyperlactatemia. ${ }^{4}$ Lactate is produced in the metabolism of glucose (Figure 1). Glycolysis converts glucose into 2 molecules of pyruvate with the net gain of 2 adenosine triphosphates (ATPs). More ATP can be generated in the oxidative part of the glucose metabolism: by entrance of pyruvate to the tricarboxylic acid cycle (TCA or Krebs cycle) and the oxidative phosphorylation in several organs like the liver, kidneys, and muscles. Pyruvate is the molecule that links glycolysis to TCA and oxidative phosphorylation. Pyruvate can be converted to lactate by a reversible oxido-reduction reaction catalyzed by the enzyme lactate dehydrogenase in the cytosol (Figure 1). Under normal

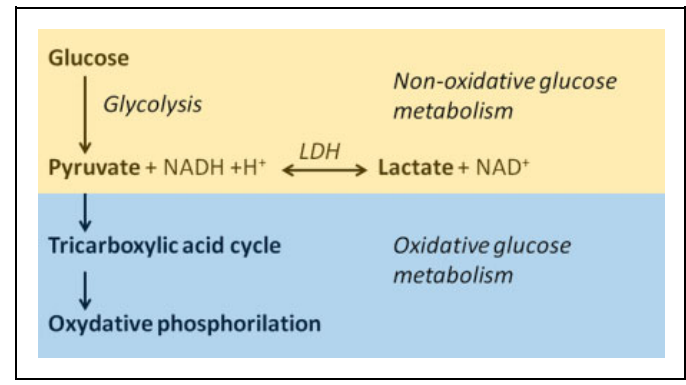

Figure I. Nonoxidative and oxidative glucose metabolism.

circumstances, TCA and oxidative phosphorylation provide the major amount of ATP for cellular function. The rate of glycolysis is faster than the TCA and oxidative phosphorylation, as a consequence glycolysis can briefly provide far more ATP in situations of cellular stress. Excess pyruvate will rapidly accumulate and is converted into lactate. In case large amounts of

\footnotetext{
'Department of Pulmonology and Critical Care, Langone Medical Center, Bellevue Hospital, New York University, New York, NY, USA

${ }^{2}$ Department of Intensive Care Adults, Erasmus MC University Medical Center, Rotterdam, the Netherlands

${ }^{3}$ Division of Pulmonary, Allergy and Critical Care, Columbia University College of Physicians and Surgeons, University Medical Center, New York, NY, USA

${ }^{4}$ Departamento de Medicina Intensiva, Facultad de Medicina, Pontificia Universidad Católica de Chile, Santiago, Chile
}

Received February 02, 2017. Received revised April 03, 2017. Accepted for publication April 17, 2017.

\section{Corresponding Author:}

Eva E. Vink, Department of Pulmonology and Critical Care, Langone Medical Center, Bellevue Hospital, New York University, New York, NY, USA.

Email: eva.e.vink@gmail.com 
Journal of Intensive Care Medicine $X X(X)$

energy are rapidly required, for example, cellular stress, lactate serves as a critical buffer that allows glycolysis to accelerate. ${ }^{5}$ Normally the lactate:pyruvate ratio is 10:1; however, when the cellular redox state changes because of anaerobic metabolism (ie, when the NADH:NAD ${ }^{+}$ratio increases), this ratio rises. ${ }^{4}$

Approximately $20 \mathrm{mmol}$ of lactate per kilogram of body weight is generated each day, mostly in the muscles and skin. ${ }^{4,6}$ Production of lactate by glycolysis is accompanied by the production of protons from the ATP degradation: glucose +2 $($ ADP + inorganic phosphate $) \rightarrow 2$ lactate $+2 \mathrm{H}^{+}+2$ ATP. As lactate consumption removes protons, the overall effect on acid-base balance is limited. ${ }^{4}$

Lactate produced by the muscles in the conversion of glycogen to glucose in anaerobic glycolysis is converted back to glycogen and glucose by the liver in gluconeogenesis. This process is called the Cori cycle and is especially important during intensive exercise. ${ }^{4}$

Normally the production and consumption of lactate are balanced. When production exceeds consumption, hyperlactatemia is the result. In combination with the accompanied production of protons, hyperlactatemia potentially results in acidosis. In clinical practice, increasing lactate levels above $5 \mathrm{mmol} / \mathrm{L}$ are frequently associated with worsening acidosis. ${ }^{7}$ Traditionally, 2 types of hyperlactatemia have been clinically used: hypoxic and nonhypoxic causes. However, both types usually coexist. Hypoxic-related causes are a result of inadequate microvascular oxygen supply. Both experimental and clinical studies have shown that a reduction in global oxygen delivery will ultimately result in a decrease in oxygen consumption. When oxygen demand remains stable, this decrease in oxygen consumption hallmarks the occurrence of tissue hypoxia and is associated with a sharp increase in lactate levels. ${ }^{8,9}$ Microcirculatory dysfunction resulting in changes in the microcirculation is also thought to play an important role. Heterogeneity of microcirculatory blood flow, varying from tissue zones receiving adequate perfusion through capillaries with continuous flow to zones without microvascular perfusion, results in impaired oxygen delivery at cellular level. ${ }^{10-12}$

In nonhypoxic causes of hyperlactatemia, increased lactate levels are the result of increased glycolysis depending on other factors than hypoxia as a result of metabolic changes, impaired clearance of lactate, or toxic causes. There are multiple metabolic causes for hyperlactatemia, for example, a cytokinedependent increase of cellular glucose uptake in inflammatory states, mitochondrial dysfunction, drugs that impair oxidative phosphorylation, and stimulation by epinephrine. ${ }^{4}$ Experimental data suggest that epinephrine induces hyperlactatemia by binding to muscle adrenergic $\beta 2$-receptors and raising AMP production, ${ }^{13}$ leading in turn to the concurrent stimulation of both $\mathrm{Na}^{+}-\mathrm{K}^{+}$-ATPase activity and glycogenolysis. ${ }^{14}$ Activation of $\mathrm{Na}^{+}-\mathrm{K}^{+}$-ATPase generates ADP and liberates $\mathrm{H}+$, thereby raising phosphofructokinase activity, accelerating aerobic glycolysis, and thus increasing lactate concentration and decreasing $\mathrm{pH}$.

Impaired lactate clearance may contribute to hyperlactatemia, although studies show conflicting results. Levraut et al
Table I. Potential Use of Lactate as a Diagnostic Test.

\begin{tabular}{|c|c|}
\hline Situation & Pathophysiology \\
\hline Grand mal seizures & $\begin{array}{l}\text { Excessive muscle activity resulting in increased } \\
\text { anaerobic glycolysis. Hyperlactatemia is } \\
\text { transient and should resolve quickly after } \\
\text { cessation of the seizures. Sustained } \\
\text { hyperlactatemia after seizure suggests } \\
\text { another or concomitant origin. }\end{array}$ \\
\hline Lymphoma & $\begin{array}{l}\text { The Warburg effect: hyperlactatemia as a result } \\
\text { of aerobic production of lactate as a source } \\
\text { of energy. }\end{array}$ \\
\hline HIV medication & $\begin{array}{l}\text { Nucleoside reverse transcriptase inhibitors } \\
\text { interfere with oxidative phosphorylation. }\end{array}$ \\
\hline Metformin & $\begin{array}{l}\text { Hyperlactatemia is usually seen in high } \\
\text { metformin plasma levels. Metformin } \\
\text { interferes with oxidative phosphorylation as } \\
\text { it suppresses hepatic gluconeogenesis. }\end{array}$ \\
\hline Metabolic diseases & $\begin{array}{l}\text { Rare inborn errors of metabolism can cause } \\
\text { dysfunction in a variety of metabolic steps } \\
\text { including gluconeogenesis, pyruvate } \\
\text { dehydrogenase, the tricarboxylic acid cycle, } \\
\text { and the respiratory chain. }\end{array}$ \\
\hline $\begin{array}{l}\text { Asthma } \\
\text { exacerbation }\end{array}$ & $\begin{array}{l}\text { Increased muscle activity resulting in augmented } \\
\text { anaerobic glycolysis and (excessive) use of } \\
\beta_{2} \text {-sympathicomimetics resulting in } \\
\text { stimulation of aerobic glycolysis. }\end{array}$ \\
\hline Thiamin deficiency & Impairment of pyruvate dehydrogenase activity. \\
\hline Liver dysfunction & $\begin{array}{l}\text { The liver accounts for up to } 70 \% \text { of whole-body } \\
\text { lactate clearance. Chronic liver diseases } \\
\text { rarely generate hyperlactatemia but } \\
\text { augments it in case of sepsis. Hyperlactatemia } \\
\text { is, however, common in acute liver failure. }\end{array}$ \\
\hline $\begin{array}{l}\text { Carbon monoxide } \\
\text { poisoning }\end{array}$ & $\begin{array}{l}\text { Decreased oxygen delivery to the tissues and } \\
\text { interference with oxidative phosphorylation. }\end{array}$ \\
\hline $\begin{array}{l}\text { Severe anemia } \\
\text { Pheochromocytoma }\end{array}$ & $\begin{array}{l}\text { Decreased oxygen delivery to the tissues. } \\
\text { Induced } \beta 2 \text {-adrenoceptor stimulation due to } \\
\text { high levels of catecholamines. }\end{array}$ \\
\hline
\end{tabular}

demonstrated that in patients with sepsis, despite hemodynamically stable and normal liver function, lactate clearance can be reduced possibly through inhibition of pyruvate dehydrogenase. ${ }^{15}$ On the other hand, Revelly et al showed that hyperlactatemia was mainly related to increased production in patients with sepsis, septic shock, or cardiogenic shock. Lactate clearance in these patients was comparable to healthy patients. ${ }^{16}$ In a recent experimental study, Tapia et al showed that clearance of lactate by the liver was almost abolished during septic shock conditions. ${ }^{17}$

Finally, multiple drugs influence lactate levels. Metformin can cause hyperlactatemia due to interference with oxidative phosphorylation as it suppresses hepatic gluconeogenesis. Hyperlactatemia is usually seen in high metformin plasma levels. $\beta_{2}$-agonists can cause hyperlactatemia by stimulating aerobic glycolysis. Moreover, nucleoside reverse transcriptase inhibitors interfere with oxidative phosphorylation potentially resulting in hyperlactatemia. ${ }^{4,8}$ As a consequence, hyperlactatemia can be present in all different types of diseases and can be a helpful measurement in the diagnostic process (Table 1). 
In summary, hyperlactatemia represents an imbalance between increased production in the presence or absence of tissue hypoxia and changes in clearance.

\section{Lactate as Predictor of Outcome}

The prognostic value of multiple types of lactate levels or calculations from lactate levels has been investigated in diverse groups of critically ill patients, for example, initial lactate levels at presentation, duration of hyperlactatemia, the area under the receiver operating characteristic (AUROC) curve, and lactate clearance (the initial lactate - subsequent lactate/initial lactate $\times 100 \%$ ). The use of this former definition is confusing, as lactate clearance should be defined as the metabolism of lactate. ${ }^{18}$ Therefore, we recommend to use the term change or decrease in lactate, since it reflects the end result of both lactate production and consumption.

The predictive value of the initial lactate level has been investigated in diverse populations, for example, in septic patients, ${ }^{19-23}$ general ICU patients, ${ }^{24,25}$ critically ill patients, ${ }^{26}$ patients with a pulmonary embolism ${ }^{27}$ and patients admitted to the ICU after high-risk surgery. ${ }^{28}$ In all these groups of patients, the initial lactate level is a predictor of outcome. Studies in trauma patients show, however, mixed results. ${ }^{29-32}$

In a heterogeneous ICU population, the duration of hyperlactatemia is related to sequential organ failure assessment scores and its organ subscores as an indicator of organ failure/dysfunction. The respiratory and coagulation subscores were most strongly associated with hyperlactatemia. This relationship was stronger during the early phase of ICU stay. ${ }^{25}$ In patients with septic shock at the ICU, ${ }^{33,34}$ trauma patients, ${ }^{35}$ and a mixed population of ICU patients, ${ }^{36}$ decreasing lactate levels or normalization of lactate are related with better outcomes. In contrast, in a heterogeneous population of patients with different causes of low-oxygen transport (eg, hemorrhage, low cardiac output, low hemoglobin level, or oxygen saturation), only the lactate levels at admission, but not the reduction over time, predicted mortality. ${ }^{33}$

The AUROC curve gives information on the accuracy of a test. The accuracy of lactate to predict mortality in the ED and ICU varies from moderate $(0.53)$ to excellent $(0.99)$. Shapiro et al found that lactate at admission in patients with suspected infections had an AUROC curve of 0.67. ${ }^{22}$ Kaplan and Kellum showed the initial lactate level to have an AUROC curve of 0.99 in trauma patients. ${ }^{32}$ In the ICU, the AUROC curve varied from 0.53 in hemodynamically unstable critically ill patients ${ }^{37}$ and 0.58 in hemodynamically stable patients after high-risk surgery ${ }^{28}$ to 0.86 in patients after hepatectomy. ${ }^{38}$ In these heterogeneous groups of critically ill patients, measured lactate shows varied prognostic performance.

In patients with a cardiac arrest, the change in lactate levels is related to mortality. Higher decreases in lactate at 6 and 12 hours are associated with decreased mortality (both 24 hours and in-hospital mortality). ${ }^{39}$ In patients who survived the first 48 hours after cardiac arrest, lactate at 48 hours is an independent predictor of mortality and unfavorable neurologic outcome. Persistent hyperlactatemia over 48 hours predicted a poor prognosis. ${ }^{40}$ Also patients with sepsis having a successful decrease in lactate (defined as a decrease in lactate $\geq 10 \%$ ) show higher survival rates compared with patients in whom lactate levels decreased less than $10 \%{ }^{41,42}$ Puskarich et al identified early lactate normalization (defined as a decrease in lactate to $2.0 \mathrm{mmol} / \mathrm{L}$ ) during the first 6 hours of resuscitation as the strongest independent predictor of survival and superior to other measures of lactate kinetics. ${ }^{23}$ Nguyen et al showed that in patients with severe sepsis or septic shock, there was a $\pm 11 \%$ decreased likelihood of mortality for each $10 \%$ decrease in lactate. ${ }^{42}$

In a recent meta-analysis of 96 clinical studies, Vincent et al showed that a decrease in lactate levels was associated with improved outcome in almost all subgroups of critically ill patients, ${ }^{43}$ thereby acknowledging the universal predictive power of lactate levels. Despite this long-standing and strong predictive power of increased lactate levels to predict increased morbidity and mortality, no studies have been published on the clinical use of this characteristic. In a study by Jansen et al on the use of lactate to guide initial resuscitation, even patients with a $100 \%$ predicted mortality based on admission lactate levels were admitted. ${ }^{3}$ Up until now, lactate levels and the association with mortality have only been used to advocate aggressive treatment, ${ }^{44,45}$ hereby ignoring the complex origin of increased lactate levels in critically ill patients. ${ }^{46}$

In summary, despite its long-standing predictive power, it is still unclear how lactate levels influence clinical decisionmaking with regard to admission policies or end-of-life decisions.

\section{Lactate as a Marker to Initiate Therapy}

As mentioned above, increased lactate levels have been advocated to initiate treatment in many clinical conditions. As vital signs can be misleading in the identification of patients with circulatory dysfunction, as for example, in early phases of shock,${ }^{47}$ increased lactate levels have been used to characterize patients with impaired tissue perfusion and oxygenation. ${ }^{48,49}$ Indeed, when one relies on vital signs only to identify patients with inadequate perfusion, a substantial number of patients with prognostically important hypoperfusion may remain undetected. ${ }^{50,51}$ This has been demonstrated for patients with cardiogenic shock, ${ }^{51}$ trauma patients, ${ }^{50}$ hemodynamic stable patients in the ICU after high-risk surgery, ${ }^{28}$ and critically ill patients in the ED. ${ }^{52}$ Only when compensatory mechanisms fail, vital signs will change. Delayed identification of patients with compensated shock may lead to delayed or inadequate resuscitation. Ideally, disease severity is already assessed before presentation at the ED. Several studies demonstrated the potential use of prehospital lactate sampling as a warning signal to initiate treatment. Prehospital lactate was significantly associated with emergent operations and multiple organ dysfunction in trauma patients. ${ }^{29}$ Compared to systolic blood pressure, lactate was a better predictor for the need of significant packed red blood cell transfusion in trauma patients with a 
relatively normal systolic blood pressure $(90-110 \mathrm{~mm} \mathrm{Hg})$ at presentation. ${ }^{53}$ Moreover, van Beest et al showed that significantly more patients with shock and high ( $>4 \mathrm{mmol} / \mathrm{L}$ ) lactate levels needed intubation compared to patients with shock and low $(<4 \mathrm{mmol} / \mathrm{L})$ lactate levels $(1.4 \%$ vs $24.6 \%){ }^{54}$

However, very few studies have reported on the efficacy of actually using lactate levels to guide treatment. In cardiac surgery patients, Polonen et al studied the effect of improving hemodynamics whenever mixed venous oxygenation or lactate levels were or became abnormal in a randomized study. ${ }^{55}$ Although there was no effect on mortality, the use of this protocol to initiate treatment was associated with a decrease in organ failure. ${ }^{55}$ In a mixed group of patients with circulatory failure (defined as a lactate level $\geq 3.0 \mathrm{mmol} / \mathrm{L}$ ), Jansen et al investigated lactate-guided treatment. ${ }^{3}$ Patients were randomized to either treatment guided by lactate levels with the objective to decrease lactate by $\geq 20 \%$ per 2 hours for the initial 8 hours of ICU stay or standard care without any information on lactate levels except for the initial lactate levels used to randomize the patient. The lactate group received more fluids and vasodilators in the 8-hour study period but less fluids in the follow-up period, a finding consistent with the landmark study on early goal-directed therapy of Rivers et $\mathrm{al}^{56}$ Surprisingly, there were no differences in lactate levels at any time point during the study period between both groups. This underscores the complex etiology of lactate levels in critically ill patients. Moreover, the effect of the Rivers study ${ }^{56}$ on initial resuscitation to correct tissue hypoperfusion obviously affected therapeutic measures in the control group. Nevertheless, the lactate group had a significant lower morbidity and hospital mortality. ${ }^{3}$ Therefore, it seems that lactate in addition to its use in initiating treatment may serve as a warning signal to the treatment team to rethink diagnostic and therapeutic options.

Treatment of hyperlactatemia should relate to its pathophysiology. In case of impaired tissue perfusion/oxygenation, therapeutic measures differ greatly when compared to intoxication, as for instance in metformin intoxication, or when increased lactate levels are the result of decreased clearance or increased aerobic lactate production. Given the significant effect of inadequate tissue perfusion on outcome, increased lactate levels should first be evaluated in this context. When the likelihood of tissue hypoperfusion is real, increased lactate levels should be seen as an indication to improve tissue perfusion. Crystalloid and colloid solutions are both effective in restoring perfusion in case of hypovolemia and sepsis. ${ }^{57}$ Vasopressors and inotropic agents should be administered as needed. ${ }^{49}$ It is important to take into account that the use of epinephrine is associated with increases in lactate not related to tissue hypoperfusion but increased glycolysis, glycogenolysis, and stimulation of the Na-K-pump. ${ }^{58,59}$ The balance between oxygen delivery and oxygen demand can be improved by ameliorating oxygen delivery by packed red blood cell transfusions when indicated, augmenting cardiac output, increasing $\mathrm{SaO}_{2}$ by intubation when necessary and improving microcirculatory perfusion by enhancing regional blood flow with, for example, dobutamine, enoximone, or nitroglycerin or by decreasing oxygen demand by mechanical ventilation. ${ }^{60,61}$

A meta-analysis ${ }^{62}$ and several other studies have now shown the efficacy of using increased lactate levels to initiate specific treatment aimed to improve tissue perfusion/oxygenation in critically ill patients. However, given the complex origin of increased lactate levels, this probably refers to only the first hours of ICU resuscitation. ${ }^{63}$

In summary, increased lactate levels have a prominent place in the early goal-directed therapy in critically ill patients, whereas the effects later on during ICU admission are unknown.

\section{Lactate to Monitor Adequacy of Initiated Therapies}

The Surviving Sepsis Campaign recommends the use of central venous pressure (CVP), mean arterial pressure (MAP), urine output, and central venous oxygen saturation $\left(\mathrm{ScvO}_{2}\right)$ as resuscitation goals. Targeting resuscitation to normalize lactate in patients with elevated lactate levels is recommended. ${ }^{49}$ Jones et al compared lactate decrease (target: lactate decrease $\geq$ $10 \%$ ) with $\mathrm{ScvO}_{2}$ improvement (target: $\mathrm{ScvO}_{2} \geq 70 \%$ ) as goals for sepsis resuscitation in a noninferiority trial including patients with severe sepsis and evidence of hypoperfusion or septic shock admitted to the ED. Patients were randomly assigned to one of the protocols for a maximum of 6 hours. Administered treatments did not differ between the groups, and there were no differences in in-hospital mortality. ${ }^{64}$ This suggests that lactate measurements perform as good as $\mathrm{ScvO}_{2}$ measurements. However, this study has some severe limitations to draw such conclusions. At first, it is questionable whether a $10 \%$ reduction in lactate in 6 hours represents effective resuscitation. Moreover, $\mathrm{ScvO}_{2}$ measurements can potentially give insight into the pathogenesis of hyperlactatemia by differentiating between hypoxic and nonhypoxic causes of hyperlactatemia. Additionally, only $10 \%$ of the patients received either dobutamine or packed red blood cell transfusions. Finally, fluids and vasopressors were guided by CVP and MAP in both groups. As a result, the potential difference in protocol actions directly attributable to either lactate or $\mathrm{ScvO}_{2}$ measurements was very small. Therefore, it seems unlikely that a change in this resuscitation target could increase mortality by $10 \%$, the noninferiority margin selected for the trial..$^{5}$

In a substudy of the Jones study, Puskarich et al demonstrated in patients with septic shock undergoing early resuscitation in the ED that failure to achieve the targeted lactate decrease (lactate $<2 \mathrm{mmol} / \mathrm{L}$ within 6 hours) was associated with worse prognosis than failure to achieve the $\mathrm{ScvO}_{2}$ goal $\left(\mathrm{ScvO}_{2} \geq 70 \%\right){ }^{65}$

Hernandez et al investigated the time course of lactate normalization during successful resuscitation. ${ }^{63}$ They suggested that the normalization of lactate consists of 2 phases: an early rapid response (flow-responsive phase) and a later slower recovery trend (potentially explained by non-flow- 
dependent mechanisms). Interestingly, pure flow-dependent flow-responsive variables such as $\mathrm{ScvO}_{2}$, capillary refill time, and central venous-arterial $\mathrm{pCO}_{2}$ showed a faster normalization rate than lactate. ${ }^{63}$ Therefore, the use of serial lactate levels as a clinical target is limited by the slow changes in lactate levels. They should rather be seen as regular "checks" to make sure the management is appropriate. Quick normalization of lactate levels as a resuscitation goal can potentially have negative effects like fluid overload. Therefore, it seems not adequate to use lactate levels as the only goal of resuscitation. Moreover, a substantial proportion of patients with septic shock have lactate levels within the normal range. On the other side, also in critically ill patients admitted to the ICU with relative hyperlactatemia (lactate levels $0.0-2.0 \mathrm{mmol} / \mathrm{L}$ ), high normal levels are independently associated with increased hospital mortality. ${ }^{66}$ The authors suggest that the reference values require adjustment since patients with lactate concentrations $>0.75 \mathrm{mmol} / \mathrm{L}$ are already at a higher risk of dying. ${ }^{66}$

In summary, a decrease in lactate levels following initiation of therapy is universally a good sign. An increase in lactate levels or even no change should warrant the treatment team to rethink the diagnosis and rationale of current treatment. Lactate levels should also be monitored when they are initially normal, to identify early a deterioration.

\section{Conclusion}

The pathophysiology of hyperlactatemia is complex with hypoxic and nonhypoxic mechanisms, often coinciding. Hyperlactatemia is a strong predictor of mortality in diverse populations of critically ill patients. It is, however, still unclear how lactate levels influence clinical decision-making with regard to admission policies or end-of-life decisions. Increased lactate levels have a prominent place in the early treatment of critically ill patients, whereas the effects later on during ICU admission are unknown. A decrease in lactate levels following initiation of therapy is universally a good sign. An increase in lactate levels or even no change should warrant the treatment team to rethink the diagnosis and rationale of current treatment.

\section{Declaration of Conflicting Interests}

The author(s) declared no potential conflicts of interest with respect to the research, authorship, and/or publication of this article.

\section{Funding}

The author(s) received no financial support for the research, authorship, and/or publication of this article.

\section{References}

1. Kompanje EJ, Jansen TC, van der HB, Bakker J. The first demonstration of lactic acid in human blood in shock by Johann Joseph Scherer (1814-1869) in January 1843. Intensive Care Med. 2007; 33(11):1967-1971.

2. Broder G, Weil MH. Excess lactate: an index of reversibility of shock in human patients. Science. 1964;143(3613):1457-1459.
3. Jansen TC, van Bommel J, Schoonderbeek FJ, et al. Early lactate-guided therapy in intensive care unit patients: a multicenter, open-label, randomized controlled trial. Am J Respir Crit Care Med. 2010;182(6):752-761.

4. Kraut JA, Madias NE. Lactic acidosis. N Engl J Med. 2014; 371(24):2309-2319.

5. Bakker J, Nijsten MW, Jansen TC. Clinical use of lactate monitoring in critically ill patients. Ann Intensive Care. 2013;3(1):12.

6. Kreisberg RA. Glucose-lactate inter-relations in man. $N$ Engl $J$ Med. 1972;287(3):132-137.

7. Fall PJ, Szerlip HM. Lactic acidosis: from sour milk to septic shock. J Intensive Care Med. 2005;20(5):255-271.

8. Jansen TC, van Bommel J, Bakker J. Blood lactate monitoring in critically ill patients: a systematic health technology assessment. Crit Care Med. 2009;37(10):2827-2839.

9. Friedman G, de Backer D, Shahla M, Vincent JL. Oxygen supply dependency can characterize septic shock. Intensive Care Med. 1998;24(2):118-123.

10. Ince C. The microcirculation is the motor of sepsis. Crit Care. 2005;9(suppl 4):S13-S19.

11. Ellis CG, Bateman RM, Sharpe MD, Sibbald WJ, Gill R. Effect of a maldistribution of microvascular blood flow on capillary $\mathrm{O}(2)$ extraction in sepsis. Am J Physiol Heart Circ Physiol. 2002; 282(1):H156-H164.

12. Goldman D, Bateman RM, Ellis CG. Effect of sepsis on skeletal muscle oxygen consumption and tissue oxygenation: interpreting capillary oxygen transport data using a mathematical model. Am J Physiol Heart Circ Physiol. 2004;287(6): H2535-H2544.

13. Clausen T, Flatman JA. Beta 2-adrenoceptors mediate the stimulating effect of adrenaline on active electrogenic NaK-transport in rat soleus muscle. Br J Pharmacol. 1980; 68(4):749-755.

14. Dietz MR, Chiasson JL, Soderling TR, Exton JH. Epinephrine regulation of skeletal muscle glycogen metabolism. Studies utilizing the perfused rat hindlimb preparation. J Biol Chem. 1980; 255(6):2301-2307.

15. Levraut J, Ciebiera JP, Chave S, et al. Mild hyperlactatemia in stable septic patients is due to impaired lactate clearance rather than overproduction. Am J Respir Crit Care Med. 1998; 157(4 pt 1):1021-1026.

16. Revelly JP, Tappy L, Martinez A, et al. Lactate and glucose metabolism in severe sepsis and cardiogenic shock. Crit Care Med. 2005;33(10):2235-2240.

17. Tapia P, Soto D, Bruhn A, et al. Impairment of exogenous lactate clearance in experimental hyperdynamic septic shock is not related to total liver hypoperfusion. Crit Care. 2015; 19:188.

18. Vincent JL. Serial blood lactate levels reflect both lactate production and clearance. Crit Care Med. 2015;43(6):e209.

19. Howell MD, Donnino M, Clardy P, Talmor D, Shapiro NI. Occult hypoperfusion and mortality in patients with suspected infection. Intensive Care Med. 2007;33(11):1892-1899.

20. Trzeciak S, Dellinger RP, Chansky ME, et al. Serum lactate as a predictor of mortality in patients with infection. Intensive Care Med. 2007;33(6):970-977. 
21. Mikkelsen ME, Miltiades AN, Gaieski DF, et al. Serum lactate is associated with mortality in severe sepsis independent of organ failure and shock. Crit Care Med. 2009;37(5):1670-1677.

22. Shapiro NI, Howell MD, Talmor D, et al. Serum lactate as a predictor of mortality in emergency department patients with infection. Ann Emerg Med. 2005;45(5):524-528.

23. Puskarich MA, Trzeciak S, Shapiro NI, et al. Whole blood lactate kinetics in patients undergoing quantitative resuscitation for severe sepsis and septic shock. Chest. 2013;143(6):1548-1553.

24. Smith I, Kumar P, Molloy S, et al. Base excess and lactate as prognostic indicators for patients admitted to intensive care. Intensive Care Med. 2001;27(1):74-83.

25. Jansen TC, van BJ, Woodward R, Mulder PG, Bakker J. Association between blood lactate levels, Sequential Organ Failure Assessment subscores, and 28-day mortality during early and late intensive care unit stay: a retrospective observational study. Crit Care Med. 2009;37(8):2369-2374.

26. Jansen TC, van BJ, Mulder PG, Rommes JH, Schieveld SJ, Bakker J. The prognostic value of blood lactate levels relative to that of vital signs in the pre-hospital setting: a pilot study. Crit Care. 2008;12(6):R160.

27. Vanni S, Viviani G, Baioni M, et al. Prognostic value of plasma lactate levels among patients with acute pulmonary embolism: the thrombo-embolism lactate outcome study. Ann Emerg Med. 2013; 61(3):330-338.

28. Meregalli A, Oliveira RP, Friedman G. Occult hypoperfusion is associated with increased mortality in hemodynamically stable, high-risk, surgical patients. Crit Care. 2004;8(2):R60-R65.

29. Guyette F, Suffoletto B, Castillo JL, Quintero J, Callaway C, Puyana JC. Prehospital serum lactate as a predictor of outcomes in trauma patients: a retrospective observational study. J Trauma. 2011;70(4):782-786.

30. Pal JD, Victorino GP, Twomey P, Liu TH, Bullard MK, Harken AH. Admission serum lactate levels do not predict mortality in the acutely injured patient. J Trauma. 2006;60(3):583-587.

31. Callaway DW, Shapiro NI, Donnino MW, Baker C, Rosen CL. Serum lactate and base deficit as predictors of mortality in normotensive elderly blunt trauma patients. J Trauma. 2009;66(4): 1040-1044.

32. Kaplan LJ, Kellum JA. Initial pH, base deficit, lactate, anion gap, strong ion difference, and strong ion gap predict outcome from major vascular injury. Crit Care Med. 2004;32(5): 1120-1124.

33. Jansen TC, van Bommel J, Mulder PG, et al. Prognostic value of blood lactate levels: does the clinical diagnosis at admission matter? J Trauma. 2009;66(2):377-385.

34. Bakker J, Gris P, Coffernils M, Kahn RJ, Vincent JL. Serial blood lactate levels can predict the development of multiple organ failure following septic shock. Am J Surg. 1996;171(2):221-226.

35. Claridge JA, Crabtree TD, Pelletier SJ, Butler K, Sawyer RG, Young JS. Persistent occult hypoperfusion is associated with a significant increase in infection rate and mortality in major trauma patients. J Trauma. 2000;48(1):8-14.

36. Suistomaa M, Ruokonen E, Kari A, Takala J. Time-pattern of lactate and lactate to pyruvate ratio in the first 24 hours of intensive care emergency admissions. Shock. 2000;14(1):8-12.
37. Marik PE, Bankov A. Sublingual capnometry versus traditional markers of tissue oxygenation in critically ill patients. Crit Care Med. 2003;31(3):818-822.

38. Watanabe I, Mayumi T, Arishima T, et al. Hyperlactemia can predict the prognosis of liver resection. Shock. 2007;28(1):35-38.

39. Donnino MW, Miller J, Goyal N, et al. Effective lactate clearance is associated with improved outcome in post-cardiac arrest patients. Resuscitation. 2007;75(2):229-234.

40. Kliegel A, Losert H, Sterz F, et al. Serial lactate determinations for prediction of outcome after cardiac arrest. Medicine (Baltimore). 2004;83(5):274-279.

41. Arnold RC, Shapiro NI, Jones AE, et al. Multicenter study of early lactate clearance as a determinant of survival in patients with presumed sepsis. Shock. 2009;32(1):35-39.

42. Nguyen HB, Rivers EP, Knoblich BP, et al. Early lactate clearance is associated with improved outcome in severe sepsis and septic shock. Crit Care Med. 2004;32(8):1637-1642.

43. Vincent JL, Silva QE, Couto L Jr, Taccone FS. The value of blood lactate kinetics in critically ill patients: a systematic review. Crit Care. 2016;20(1):257.

44. Casserly B, Phillips GS, Schorr C, et al. Lactate measurements in sepsis-induced tissue hypoperfusion: results from the Surviving Sepsis Campaign database. Crit Care Med. 2015;43(3):567-573.

45. Zhang $\mathrm{Z}, \mathrm{Xu} \mathrm{X}$. Lactate clearance is a useful biomarker for the prediction of all-cause mortality in critically ill patients: a systematic review and meta-analysis. Crit Care Med. 2014;42(9): 2118-2125.

46. Bakker J. Lost in translation: on lactate, hypotension, sepsis-induced tissue hypoperfusion, quantitative resuscitation and Surviving Sepsis Campaign bundles. Crit Care Med. 2015; 43(3):705-706.

47. Bakker J, Jansen TC. Don't take vitals, take a lactate. Intensive Care Med. 2007;33(11):1863-1865.

48. Cecconi M, De BD, Antonelli M, et al. Consensus on circulatory shock and hemodynamic monitoring. Task force of the European Society of Intensive Care Medicine. Intensive Care Med. 2014; 40(12):1795-1815.

49. Dellinger RP, Levy MM, Rhodes A, et al. Surviving sepsis campaign: international guidelines for management of severe sepsis and septic shock, 2012. Intensive Care Med. 2013;39(2): 165-228.

50. Eastridge BJ, Salinas J, McManus JG, et al. Hypotension begins at $110 \mathrm{~mm} \mathrm{Hg}$ : redefining "hypotension” with data. J Trauma. 2007; 63(2):291-297.

51. Ander DS, Jaggi M, Rivers E, et al. Undetected cardiogenic shock in patients with congestive heart failure presenting to the emergency department. Am J Cardiol. 1998;82(7):888-891.

52. Rady MY, Rivers EP, Nowak RM. Resuscitation of the critically ill in the ED: responses of blood pressure, heart rate, shock index, central venous oxygen saturation, and lactate. Am J Emerg Med. 1996;14(2):218-225.

53. Vandromme MJ, Griffin RL, Weinberg JA, Rue LW III, Kerby JD. Lactate is a better predictor than systolic blood pressure for determining blood requirement and mortality: could prehospital measures improve trauma triage? J Am Coll Surg. 2010;210(5): 861-869. 
54. van Beest PA, Mulder PJ, Oetomo SB, van den BB, Kuiper MA, Spronk PE. Measurement of lactate in a prehospital setting is related to outcome. Eur J Emerg Med. 2009;16(6):318-322.

55. Polonen P, Ruokonen E, Hippelainen M, Poyhonen M, Takala J. A prospective, randomized study of goal-oriented hemodynamic therapy in cardiac surgical patients. Anesth Analg. 2000;90(5): 1052-1059.

56. Rivers E, Nguyen B, Havstad S, et al. Early goal-directed therapy in the treatment of severe sepsis and septic shock. $N$ Engl J Med. 2001;345(19):1368-1377.

57. Severs D, Hoorn EJ, Rookmaaker MB. A critical appraisal of intravenous fluids: from the physiological basis to clinical evidence. Nephrol Dial Transplant. 2015;30(2):178-187.

58. Day NP, Phu NH, Bethell DP, et al. The effects of dopamine and adrenaline infusions on acid-base balance and systemic haemodynamics in severe infection. Lancet. 1996;348(9022): 219-223.

59. Levy B, Mansart A, Bollaert PE, Franck P, Mallie JP. Effects of epinephrine and norepinephrine on hemodynamics, oxidative metabolism, and organ energetics in endotoxemic rats. Intensive Care Med. 2003;29(2):292-300.

60. Hernandez G, Pena H, Cornejo R, et al. Impact of emergency intubation on central venous oxygen saturation in critically ill patients: a multicenter observational study. Crit Care. 2009; 13(3):R63.

61. Aubier M, Viires N, Syllie G, Mozes R, Roussos C. Respiratory muscle contribution to lactic acidosis in low cardiac output. Am Rev Respir Dis. 1982;126(4):648-652.

62. Gu WJ, Zhang Z, Bakker J. Early lactate clearance-guided therapy in patients with sepsis: a meta-analysis with trial sequential analysis of randomized controlled trials. Intensive Care Med. 2015; 41(10):1862-1863.

63. Hernandez G, Luengo C, Bruhn A, et al. When to stop septic shock resuscitation: clues from a dynamic perfusion monitoring. Ann Intensive Care. 2014;430.

64. Jones AE, Shapiro NI, Trzeciak S, Arnold RC, Claremont HA, Kline JA. Lactate clearance vs central venous oxygen saturation as goals of early sepsis therapy: a randomized clinical trial. JAMA. 2010;303(8):739-746.

65. Puskarich MA, Trzeciak S, Shapiro NI, et al. Prognostic value and agreement of achieving lactate clearance or central venous oxygen saturation goals during early sepsis resuscitation. Acad Emerg Med. 2012;19(3):252-258.

66. Nichol AD, Egi M, Pettila V, et al. Relative hyperlactatemia and hospital mortality in critically ill patients: a retrospective multicentre study. Crit Care. 2010;14(1):R25. 